\title{
The Potentials for Hands-Free Interaction in Micro-Neurosurgery
}

\author{
Hoorieh Afkari \\ School of Computing \\ University of Eastern Finland, \\ Finland \\ hoorieh.afkari@uef.fi
}

\author{
Shahram Eivazi \\ School of Computing \\ University of Eastern Finland, \\ Finland \\ shahram.eivazi@uef.fi \\ Susanne Mäkelä \\ School of Computing \\ University of Eastern Finland, \\ Finland \\ susanne.makela@uef.fi
}

\author{
Roman Bednarik \\ School of Computing \\ University of Eastern Finland, \\ Finland \\ roman.bednarik@uef.fi
}

\begin{abstract}
Micro-neurosurgery has been revolutionized by advances in the surgical microscope such as high magnification that have increased a surgeon's ability to have a clear view of the surgical field. High magnification necessitates frequent interaction with the microscope during an operation, and the current interaction technique for positioning and adjusting the microscope introduces risk factors that force a surgeon to remove hands from the operating field. The purpose of this study is to investigate the potential for hands-free interaction in microneurosurgery. We present findings from a contextual study of how neurosurgeons interact with the microscope and the surgical team, and discuss the implications of the findings for designing hands-free, especially gaze-based interaction techniques for micro-neurosurgery.
\end{abstract}

\section{Author Keywords}

Medical practice; microscope; operating theater; neurosurgery; contextual study; hands-free interaction; eye-tracking.

\section{ACM Classification Keywords}

H.5.m. Information Interfaces and Presentation (e.g. HCI): Miscellaneous

\section{INTRODUCTION}

Over past decades, healthcare technologies have taken advantage of innovations from imaging and human-computer interaction (HCI) research to improve diagnosis, treatment, and patient safety. Micro-surgery, particularly microneurosurgery, is one of the medical domains that has made

Permission to make digital or hard copies of all or part of this work for personal or classroom use is granted without fee provided that copies are not made or distributed for profit or commercial advantage and that copies bear this notice and the full citation on the first page. Copyrights for components of this work owned by others than ACM must be honored. Abstracting with credit is permitted. To copy otherwise, or republish, to post on servers or to redistribute to lists, requires prior specific permission and/or a fee. Request permissions from Permissions@ acm.org. NordiCHI '14, October 26 - 30 2014, Helsinki, Finland Copyright 2014 ACM 978-1-4503-2542 4/14/10.15.00.http : //dx.doi.org/10.1145/2639189.2639213 great strides in improving surgical outcomes using these new devices. The procedure of micro-neurosurgery is a delicate combination of various techniques and tools with complex interactions among the team members and between the tools and the users [10, 23, 28]. This surgery is performed on micro-scale structures of the nervous system under a high magnification using a surgical microscope and miniaturized instruments.

It took about forty years from the first microscope use in laboratory medical research to the first micro-neurosurgery [40]. Today, regardless of the surgery type and steps, neurosurgeon uses the microscope almost during the entire operation (as suggested by expert [10]). This intensive interaction with a microscope is the core of interest for this research.

To date, the development and research into the operation of a surgical microscope has focused on two primary principles: 1) the stereoscopic magnified view of the anatomical structures to improve the operating precision and prevent errors $[25,35,40]$, and 2) the freedom to smoothly operate, move, and otherwise adjust the microscope to keep the surgery simple and fast $[3,10,35]$. From the technological point of view, the present surgical microscopes provide clear magnification with powerful illumination. A sharp view of the operating field, which is the result of a controlled zoom and focus system, enables surgeons to have natural perception of the surgical field [20]. In addition, the option to adjust the position of the whole instrument allows a surgeon to select different positions as required. What we will show later in this work, is that the human-computer interaction principles have not been carefully taken into account along the strides of the technology development.

We will show in detail that although high magnification is an important factor for the success of many complex surgical procedures, it has some significant disadvantages, consequences and implications for designing interaction [26]. Operating under high magnification means smaller view field and introduces the need to maintain a clear view of all the ar- 
eas of interest by adjusting and moving the microscope. It is also not considered feasible for an assistant to adjust the microscope setting while the neurosurgeon keeps his/her eyes engaged with the microscopes eyepiece. Currently, handbased interaction techniques for adjusting a surgical microscope such as handgrip, knobs, or buttons require that surgeons remove their hands from the operative field. These practices are not risk-free and lengthen the operating time.

In response to these limitations, previous research suggested several remedies. First, a number of new input techniques such as foot switch, voice, and eye control have been suggested earlier by $[4,35,41]$ to expand the ways in which surgeons interact with the operating microscope. Our interest is also to explore possibilities of new interaction techniques for the microscope. Alongside these techniques, other types of robotic systems have been introduced into neurosurgery [18, 26]. Notwithstanding the importance of robotic techniques, developing such systems would require a total transformation of current surgery practices, this requires investigation within the broader system of this medical work and is beyond the scope and purpose of this research. In modern surgical practice, none of the described state-of-the-art techniques and systems have been adopted or have become available commercially, thus hand control remains the status quo since the introduction of microscopes to the operating room. The reasons for the low rate of adoption of novel interactive technologies into medical practice vary. It could be that current interaction techniques have additional advantages that may be lost if they were replaced with a new input system [17]. Therefore, introduction of a new interactive technology to the operating room (OR) needs to start with a careful investigation of the design space, in order to create technologies that fit and improve interaction, but, do not interfere with what works.

\section{OPERATING ROOM AS A DESIGN CONTEXT}

Work practices in healthcare have been widely studied in HCI and computer-supported cooperative work (CSCW) [7], and many of these studies have provided insights into the activities of surgical operations [1, 17, 21, 24]. A surgery involves a complex division of labour in which people with very different skills and roles communicate and collaborate with each other. Unlike in many other types of collaborative work, in surgeries, the collaboration of the team focuses on supporting one main activity that is performed by the surgeon [31].

One of the research interests in an operating room has been how awareness [9] is produced and sustained during a surgery. A recent study by Kusunoki et al. [21] explored how vital signs monitors support decision making and situation awareness for medical teams. Awareness in OR is also mediated through the use of tools: tools can be, for example, arranged and configured during the operation to reflect the current or expected state of the procedure [31].

The studies have also highlighted how crucial the coordination of activities between a surgeon and a scrub nurse is for the success of a surgical operation [13, 31]. A scrub nurse does not only pass instruments to a surgeon: based on her knowledge and experience, she organises the instruments considering the current stage of the procedure and a surgeon's personal preferences, anticipates a surgeon's actions, and prepares the instruments accordingly. In this way, a surgeon can work fluently without interruptions and change instruments as required [28].

Related to technology use, Nardi et al. [28] have discussed the crucial role of live video for the surgical team in neurosurgeries. A video of the surgical field that would be otherwise visible only to the surgeon provides a common focus that supports shared awareness of the surgeon's work and helps the surgical team coordinate their activities. The live video is especially important for a scrub nurse for observing a surgeon's actions. In the nurse's case, however, watching of the live video is frequently interrupted by other tasks, which means that she needs to understand quickly what is going on in the video. The rest of the team often watch video for longer periods of time to see how the surgery is progressing.

The use of displays was also the focus in another study that analysed how collaborative work practices and interaction are spatially organised in neurosurgery [24]. Using the concept of interaction proxemics - how properties of interactive devices affect they way in which people spatially organise themselves with respect to technology, content and each other they discussed the needs of the surgical team related to controlling images from a distance, pointing from a distance, fine vs. coarse grained referencing, and having a better and more flexible line of sight to images during a surgery.

Micro-neurosurgery, on which we focus in this work, differs from other surgeries in its reliance on the microscope. In micro-neurosurgery, the video image comes from a microscope which is used by the surgeon during the operation. The microscope can be zoomed and focused onto the operative plane or surgeon can move the microscope to change the field of view. Operating under microscope present challenges for adjusting the microscope setting while both surgeon's hand are occupied by micro-instrument in action. For the first surgical microscopes introduced to ORs in the early 1960's, time that was used to adjust and move a microscope was estimated to be about $40 \%$ of the whole duration of microscope usage time [39]. The time required for interacting with a microscope was reduced significantly when the first counterbalance microscope was introduced to OR in 1972 [39]. Surgeons could move microscope head freely around the operating field using an electromagnetic brake system. Since that time, these devices have been used in surgery practice for several decades and generally development of new interaction techniques for operating microscope have remained experimental. In response to these developments, to date, the argument for improving operating microscope have not been well articulated within the context of micro-neurosurgery routines and practices.

In this paper, we demonstrate a contextual study of microneurosurgical operating procedure focusing on surgeons' interaction with the surgical microscope. Our interest, in particular, is to explore the potentials for novel hands-free input interaction methods with focus on gaze-based interaction. Previous studies [4] that proposed gaze interaction techniques in operating room only addressed the technical aspect of the sys- 
tem and discussed the technological implementation. However, due to the complexity of the actual practice [22, 35], to fulfill surgeons' needs and requirements, first an in-depth exploration of the design space is essential. Therefore, we take a step back to identify the design space opportunities and challenges, work flow, and communication patterns through the lenses of a field study of microscope use during microneurosurgery.

\section{FIELD STUDY IN NEUROSURGERY ENVIRONMENT}

This fieldwork profits from the contextual observation approaches as typically performed in $\mathrm{HCI} / \mathrm{CSCW}$ practice, and complements the data with video analysis, interviews and think aloud data to understand the collaboration flow in micro-neurosurgical environment. The study has been conducted at Neurosurgery of KUH NeuroCenter, Kuopio University Hospital and Institute of Clinical.

In a course of one year we observed over 20 surgeries and during two extra weeks of observation 6 surgeries were audioand video recorded. The detailed data collection was performed in two operating theaters where three brain aneurysm and three spine surgeries were performed. The average time of each surgery was about 100 minutes and recordings covered the whole surgery time. Four senior neurosurgeons performed the surgeries and two of the latest models of microscopes (Zeiss Pentero) were used during these surgeries. Surgeons were all right-handed. In one case, a short part of a surgery was conducted by a resident under supervision of the main surgeon. In addition to the recorded video materials, our data also contains the interview data of the surgeons and residents which were collected before and after each surgery. We were free to ask questions during surgeries and surgeons were encouraged to provide us explanations about the operation procedure while performing surgeries.

\section{MICRO-NEUROSURGERY THEATER SETTING}

A typical plan of a surgical operating room (OR) is showed in Figure 1. Providing fast and easy access for personnel, to patient and other equipments is the main concern in the OR setup. Maintaining a regular routines necessitates optimal arrangements of the facility, however, in case of any emergencies these spatial design factors are even more important.

The OR physical arrangement is set up to maintain the clear line of sight for all OR personnel and to respect the sterile zone during surgery. The patient table is located in the center of the room. Sets of medical equipment are positioned around the patient table. Surgeon's position dictates the location of scrub nurse and instrument table. Typically, two instrument trolleys are placed close to the patient table on the right side of the surgeon; this provides full access for both scrub nurse and surgeon. Scrub nurse has control over the instrument tables in the hand-off zone. Regardless of the surgery steps, the hand off zone between surgeon and scrub nurse must be maintained available and clear.

Situation awareness of the OR personnel is supported by projecting the microscope view from the operating field to several displays. Real time video helps anesthesiologist, nurses and other personnel to keep track of the surgery progress.

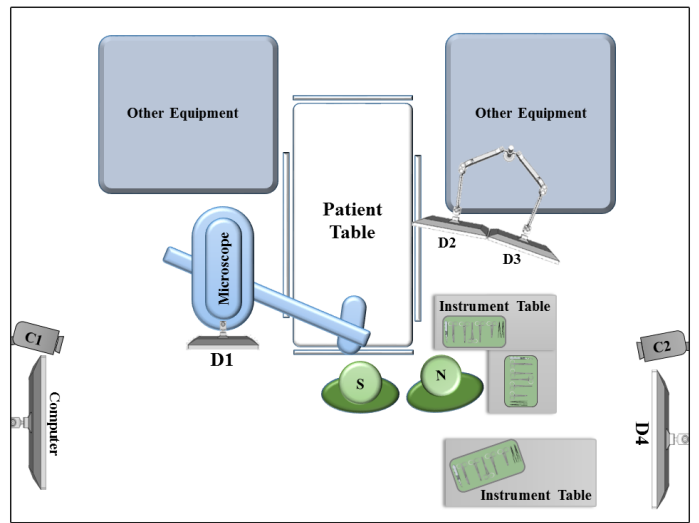

Figure 1. Typical micro-neurosurgical OR setting and configuration. It shows the position of the surgeon $(\mathrm{S})$, scrub nurse $(\mathrm{N})$, microscope, displays (D1, D2, D3, D4), cameras and tables. Among displays, D2 and D3 specifically are used by scrub nurse. D1 is microscope screen which is used by circulating nurse to activate the recording and adjust the illumination. Other OR team members follow the surgery using D4.

Surgeon has a direct access to the microscope and the body of the microscope is located on the left side of the surgeon and 2-3 meters further from patient table. In all cases, surgeons performed the surgery in standing position which provides them full mobility and freedom to move.

The other equipment around the patient table, which includes the vital signs devices, is controlled by the anesthesia team; this team sits opposite the surgeon and microscope.

\section{WORK FLOW OVERVIEW}

A typical neurosurgical practice begins with the pre-operation procedure. As the patient enters the operating room (OR), there are a series of actions such as patient positioning, draping, preparing the sterile area the instruments, and the anesthesia. These are conducted by a scrub nurse, technicians, and an anesthesia team.

It is necessary for a neurosurgeon to be present in the OR and perform the final check of the medical images, and the microscope optics and settings before their final aseptic and gloving procedures. Surgeons also load their personal profile into microscope software, which sets options based on the surgeons' preferences. Moreover, some surgeons prefer to stay in the OR while the patient is being prepared for the surgery to check the body orientation. A surgeon explained:

"It is not a part of my duty to be present and attend to patient preparation. But I prefer to check the exact orientation of the patient's body. There are many details which can help me to have more control over the operation. For example, gravity; I consider the orientation and gravity to make the dissecting procedure easier."

Once the preparation is completed, the next phase of the operation starts. From this point, the scrub nurse assists the neurosurgeon by providing appropriate instruments and maintaining the sterile environment. The initial incision and some dissection to access the surgical site do not require the microscope. 
When the operative field is ready for dissection, then the microscope is launched. The primary neurosurgeon pulls the microscope arm and adjusts the oculars to perfect the view, and if needed, the circulating nurse helps move the microscope body closer to the patient table. The circulating nurse is also responsible for adjusting the microscope light and for activating the recording function. The microscope arm and oculars which are located close to the patient bed in the sterile area, are covered with a special sterilized wrapping during the pre-operation preparation. Therefore, to interact with unsterilized parts of the microscope, surgeons always need assistance from a circulating nurse.

The majority of the operation involves dissection, during which the neurosurgeon creates a path to the target point using miniaturized instruments under the highly magnified microscopic view. While the microscope is in use, the scrub nurse follows the surgery from display screens with a live view of the operative field provided.

After the treatment is completed, the surgeon performs the wound closure procedure with or without using the microscope. After closure, the microscope is not used, while the surgeon controls the patients status, signs documents, and then leaves the OR. The remaining post-operating procedures are performed by other personnel and do not include surgical use of the microscope. In summary, the average time distribution of each surgery step is shown in Figure 2.

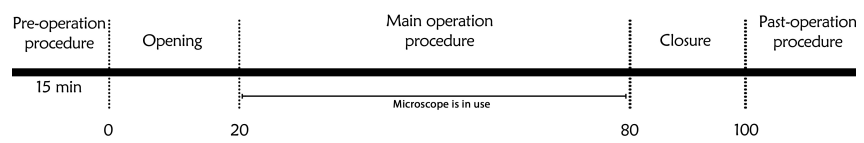

Figure 2. Average time distribution of surgeries. Microscope is used throughout the most of the surgery.

\section{INTERACTION OVERVIEW IN OR}

As the surgery progresses, various interactions occur between the clinicians themselves and also with medical devices. Two major interactions were observed during a neurosurgical practice directly relating to use of the microscope. Regardless of the type of surgery and phase, there was interaction between the surgeon and the nearest standing scrub nurse and between the surgeon and the microscope. The findings are therefore structured around these two. First, a brief description of the interaction between the scrub nurse and neurosurgeon, then details of the work practices regarding microscope use.

\section{Interaction between surgeon and scrub nurse}

The interaction between scrub nurse and surgeon starts at the beginning of the operation. We observed a great orchestration in the work between surgeon and nurse, with very few errors or distractions to the flow. The proper instruments were provided to the surgeons through a hand-off interaction while maintaining the sterile zone. A surgeon commented:

" Each surgeon follows a unique set of habits to perform the operations, therefore, scrub nurses have to be aware of the surgery routine and more importantly, the unique style of the surgeon performing an operation. This prevents loading extra works for the surgeon."

At the beginning of the operation (when the microscope is not yet in use) the scrub nurse follows the course of procedure by direct observation of the surgeons hands and the operative field. This, enables her to pass the correct instruments to the surgeon. Only a few explicit verbal requests for a particular tool were witnessed in the hand-off interaction, generally, this interaction was smooth and unproblematic without verbal discussion between surgeon and scrub nurse.

Once the operative field and microscope were ready for dissecting, the surgeons fixed the position of their eyes to the eye piece or ocular. We observed during the microscope use, for the majority of the time, the surgeons kept their eyes constantly on the ocular. Also during this dissection period, the scrub nurse had to change her view from the operative field to the OR display panel at her front. The scrub nurses only visual awareness of the procedure was at this time provided by the live view displays of the microscopes field of view (Figure $3)$.

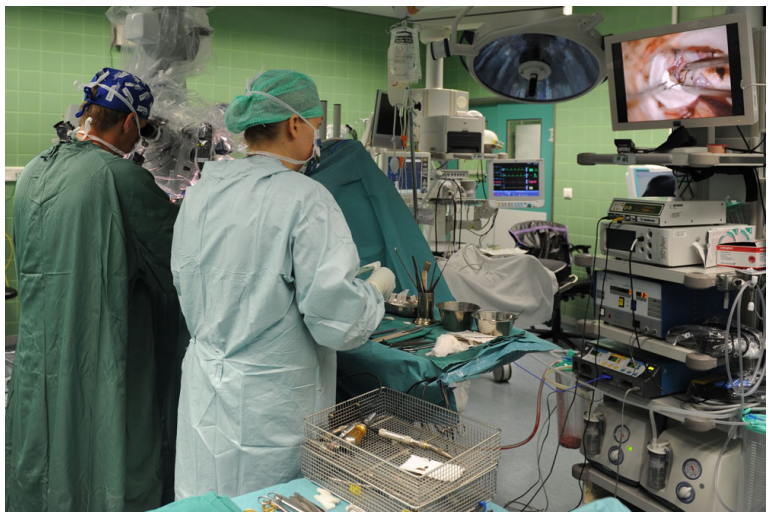

Figure 3. Scrub nurse follows the surgery by looking at the OR live view display. The display shows only a sub-area of the view the surgeon has through the oculars.

During this main part of the surgery, it is greatly important that the hand-off interaction be precise and perfectly timed, since when a surgeons eyes are positioned on the microscope oculars, there is no opportunity for him/her to look at the next instrument or the instrument table. The scrub nurse is fully responsible for putting the instruments in a surgeons hand with the correct orientation during the hand-off (Figure 4). The scrub nurse, should not only react perfectly to the surgeons needs, but she/he also has a proactive role, in which the nurse should observe the procedure and be able to predict the surgeons next move and prepare the necessary instrument. Considering the non-verbal communication observed between a surgeon and scrub nurse, the live view displays are the only source of information the nurse can rely on to act as desired. It is therefore very important to provide a scrub nurse the opportunity to maintain her/his situational awareness so that she/he can predict a surgeons next action; such opportunity is currently missing.

\section{Interaction with the surgical microscope}




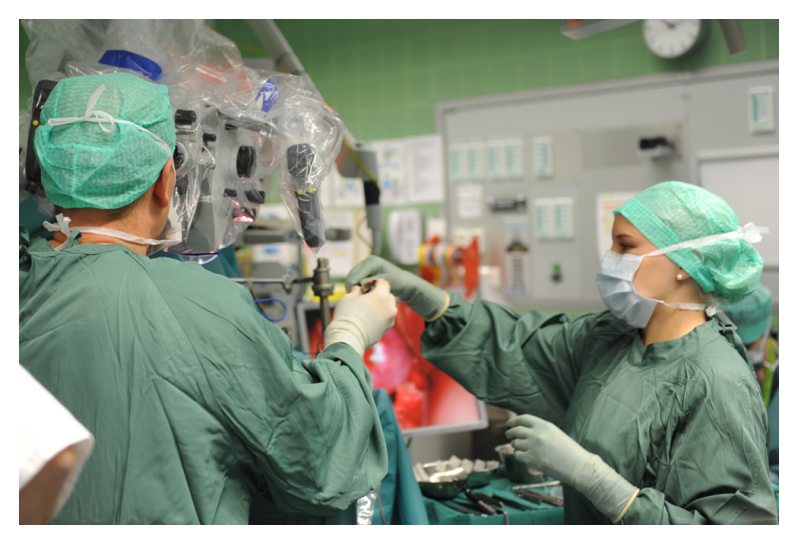

Figure 4. Hand off interaction between surgeon and scrub nurse. Surgeon does not leave the operative field unattended during handing off.

In addition to requiring a behavioral change of the scrub nurse, use of the microscope also introduced a new type of interaction to the operation. Surgeons were frequently observed having to pause their manipulation of the surgical instruments to allow them to remove a hand from the operative field so that they could interact with the microscope.

Adjusting the surgical microscope observed is possible using either handgrips or a mouth switch that is integrated into the microscopes head. Figure 5 shows one of the two handgrips which are used to move the microscope arm and head. These handles are located on the left and right side relative to the oculars.

The releasing buttons integrated into the handgrips allow the brake in the microscope arm to be controlled within a safe balanced mode, so that a surgeon can move the arm in every desired direction almost effortlessly. The grips also have control buttons and knobs for the zoom, focus, and light.

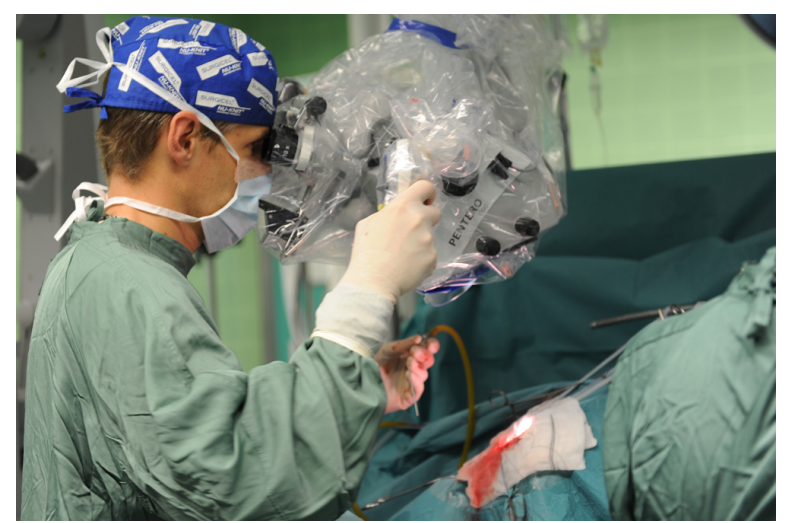

Figure 5. Surgeons interact often with the microscope handgrip during surgery. Handgrip interaction is used to adjust both the zoom, focus and the angle of the microscope.

The functionality of these buttons can be defined based on the surgeons needs and preferences and then stored in custom profiles. Another possible way to manipulate the microscope during an operation is to use a whistle-shaped mouth piece located close to the surgeon's mouth. This mouth switch has been designed to be held by a surgeons lips or teeth and includes a control for the arm brake. Figure 6 shows a current design of a mouthpiece being used to operate a microscope. This switch allows the microscope arm to be moved left and right, backward and forward, and up and down.

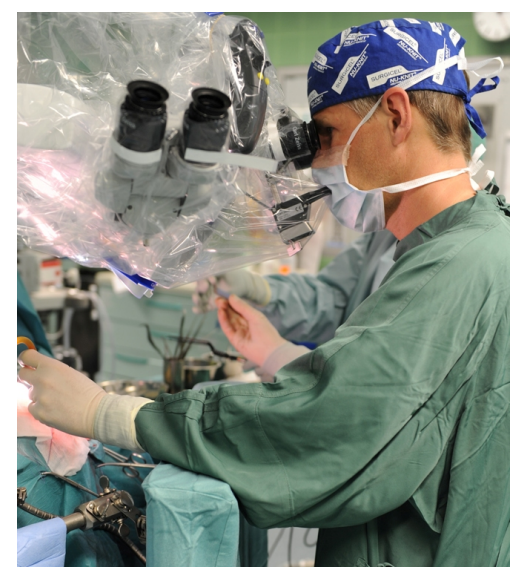

Figure 6. Mouthpiece in surgical microscope. Mouthpiece is not regarded as a usable way of interaction and its use disrupts collaborative work.

In order to maintain a clear view of the operative field surgeons were observed have to apply a set of continuous commands (e.g. moving, zooming, and focusing) manually. The frequency of these interactions varied based on the surgery type and operation phase. For example, during brain aneurysm surgeries these types of adjustment interactions were observed to be short in duration and in spatial movement. In contrast, surgeries with deeper cavities (e.g. spine surgeries) had longer and larger movements. A surgeon explained:

"The biggest adjustment to the microscope happens when we change the view direction to obtain a different perspective of the operative field."

Ideally, interaction with microscope should be smooth and uninterrupted. However, our observation shows current interactions are time-consuming and cause frequent interruptions in the ongoing procedure. Once progress in operation makes the current operative view field is out of focus or instruments go out of the view field, surgeons have to pause the operation, remove the hand(s) from the surgical field and use the handgrips to adjust the view again. Interruptions, time loss and distractions caused by these interactions lead surgeons to avoid interacting with microscope and keep performing the procedure continuously as much as possible.

"When the problematic area is not located in the surface of the brain, reaching to the desired point is a complicated procedure and if we take the hands from the field, the elasticity of brain tissue causes losing the position and forces over-work to obtain it again. Also, looking away from the microscope ocular sometimes causes loss of alignment of the operative field. "

To enable surgeons having a clear view, microscope has autofocus functionality to keep the view focused and clear. But 
we noticed this option is not being used by surgeons and they find it not usable. Frequent movements of the instruments and high magnified area make using the auto focusing not efficient. On the other hand, frequent refocusing is essential as the brain tissue is soft and distorting happens. Additionally, changing the depth of the surgical cavity necessitates constant refocusing. Hence, surgeons rely on their own surgical skills to overcome this limitation. It can be seen that surgeons are working under a blur view for some time and they do not change the zoom or focus to clarify the view field. Further, we found out that by irrigating the surgical cavity, they lose the focus and view for seconds but still they continue working and gain the clear view again.

Another approach to obtain the clear view without removing their hands from the surgical field is to use the mouth piece. This enables neurosurgeons to move and adjust the position of the microscope arm without taking the hands from the operation and thus, it is recommended by expert neurosurgeons to use mouth switch as often as possible [10]. Regardless of its advantages, we noticed that in overall, the frequency and duration of using the mouthpiece was low. By a closer look, we found the answer of why neurosurgeons do not frequently use the mouthpiece. The maneuverability provided by mouthpiece is less than handgrips as well as the movement comfort. Handgrips support movements in almost every desired direction whereas mouthpiece does not provide tilting. Moreover, controlling the suspension by mouth is harder than hands and it is often used for small and limited movements. Mouthpiece use can soak the surgeon's mask with saliva and also it is difficult to talk while mouthpiece is close to or inside the mouth. A Surgeon explained:

"Regardless of keeping the hands in the surgical field and using the mouth, still during the adjustment progress (by mouthpiece) we cannot use our hands to continue the operation. It is an interruption but at least we can use our eyes and hands to keep the position of the surgical field and maintain the situation awareness. But, the less possible movements in mouthpiece, every sometimes, forces us to remove the hands and readjust the view using the grips. It is always happening that after several small movements by mouthpiece we use grips to gain the perfect view."

Interaction with microscope including the mouthpiece is more difficult for resident due to the complex time coordination of interactions and original operation procedure. In one example, we observed how a resident received advices from a surgeon during the microscope use. The situation was highly controlled by the surgeon. Surgeon gave his position to the resident and followed the operation using the microscope coobservation ocular. The resident continued the dissecting for few minutes:

Resident: The view is not very clear anymore. I think I have to focus.

Surgeon: No, continue, it will be cleared in few seconds.

Resident: OK... [The surgical area became clear again after going few millimeters deeper.]
To further clarify our interpretation we ask a resident to explain the reasons for mentioned behavior:

"At the beginning of using the microscope, it is complicated to use our hands in one direction and following hands by eyes indirectly [Hand-eye coordinate]. Also controlling the unit by mouth needs time and experience. Surgeon delivers his experiences of using the device while controlling the surgery procedure. Here, he knew by changing the depth of the cavity the view will be cleared and he did not let me to change the focus. As we get more familiar with brain tissue and its characteristics, we know more where and when to change the view, zoom and focus."

\section{Strategies to overcome interaction problems}

The limitations related to microscope interaction affected the way surgeons manage the operation. In all of the observed operations, surgeons combine the handgrip interaction with hand off interaction. As using the grips forces the surgeon to remove the hands from the surgical field, surgeons mostly interact with microscope when they need to change the instrument. In this case, they save the time by taking the hand one time for two reasons. However, this requires the scrub nurse to have full awareness and anticipation of the exact time for adjusting the microscope.

Another example of developing an strategy to avoid interaction with microscope seen when surgeon covers the limitation of the microscope movements by having specific body postures. For example, bend their knees or stay on toes or adjusting their height by wearing or removing high-heeled clogs (Figure 7). Surgeon confirmed our observation:

"Sometimes, in order to obtain a view of the more challenging target points, we need to complete the microscope arm movements with our body postures (Figure 7)."

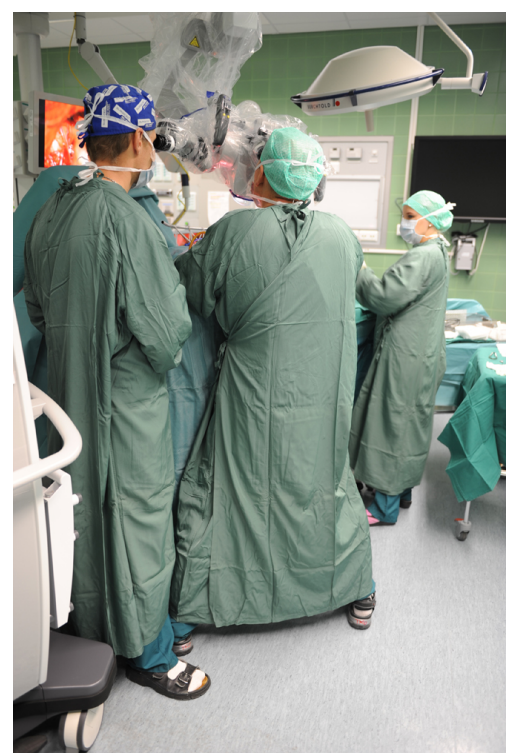

Figure 7. Poor results of microscope modalities for adjusting the unit, and avoidance to interrupt the surgery flow, lead surgeons to adopt unergonomic body postures to obtain and maintain a clear view. 
We observed avoiding interaction with microscope, brings additional risks and potential complications to the collaborative activities. Sometime surgeon had to make decision to continue operation for few seconds under unclear view or interrupt the surgery for focusing. Moreover, Often surgeons were continuing the operation when their hands reached to the border of the view field (Figure 8). This is also a hazardous situation as the the view of the microscope is wider than the live view screen of the nurse and it affects the collaborative work for scrub nurse while following the surgery.

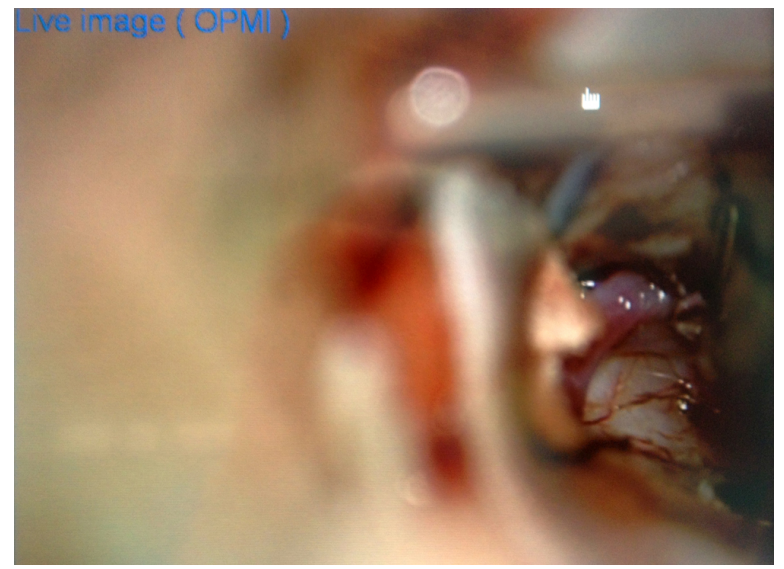

Figure 8. Microscope view is blurred and at the same time the surgeon works close to the edge of the view field.

Surgeons know if they remove hands from the surgical field or interrupt the surgery by using the mouthpiece, in order to obtain a better view they lose the time and the outcome of view change might not be as desired. Thus, they continue and adapt themselves to situation by putting effort from their own side: taking uncomfortable body postures, continue working as possible with the current view field even if it is very close to the edge of the view or unfocused, and use their surgical skills (familiarity to the body anatomy) to overcome these constrains.

\section{IMPLICATIONS FOR HANDS-FREE INTERACTION DE- SIGN}

We looked at the nuances of the microscope use in neurosurgical operating room from the perspective of interaction analysis. We have found that performing a simple, fast, and safe practice are the key objectives during a neurosurgical procedure and drives the interaction between people and medical technologies. Surgeons play a leading role in interacting with the personnel and instruments in order to expedite the progress as well as to warrant safe surgery. In order to manage critical resources effectively, surgeons make a frequent use of microscope features and seamlessly coordinate their eyes and hands within the restricted working area. Adjusting the focus and zoom, and changing the position of the microscope are the two major types of interactions.

The findings give yield to a set of requirements within the sociotechnical environment and established procedures. The requirements, we believe, can be met by various modes of hands-free interaction for surgical microscopes. With this in mind, we return to our original motivation which was to explore the potentials for new input interaction methods in neurosurgical OR settings. The general benefit of hands-free interaction techniques is that they allow the surgeons to keep their hands in the operative field while adjusting the microscope setting using the new modality. Hands-free interaction also in many cases fulfills the requirements of sterile aseptic practice.

The interaction between the leading surgeon and the assisting scrub nurse can be seen as a hallmark of flawless, interruption-free collaboration. Similarly, a new way of interacting with the surgical microscope needs to reduce the interruption of the surgery. The need for interruption reduction is supported by the observed behavior of the surgeons: they employed various strategies to keep a procedure going, including active avoidance of interaction with devices to minimize interruption. Therefore, surgeons deliberately reduce the number of times they adjust the microscope that would force them to remove hands from the operative field. Any new input modality to be applied to a microscope thus has to support uninterrupted operation. In our study, we have seen that surgeons' intention to continue working leads to unclear microscope view, to working very close to the edge of the view field, or to unergonomic positions. In many cases these strategies cannot be considered to fulfill the requirements of safe surgery.

In sum, we observed that any new interaction and change of work has to support hands-free work, minimize interruption and provide implicit way of work, allow continuous and fine grained input. The technology cannot force the users to assume unergonomic positions.

Not surprisingly, the need for a hands-free interaction technique has been a central concern of surgical microscope development since its invention. However, there are only a limited number of candidates for such a modality of control. In the following, we evaluate the feasibility of four input techniques, namely, mouth-operated input, foot control, voice input, and gaze input; Table 1 shows a comparison of these different input techniques with respect to the primary requirements.

In the early 1970s, the mouthpiece was introduced to surgical microscopes as the most practical interaction technique for repositioning or refocusing $[35,39]$. Controlling microscope with mouth enhances the mobility of the microscope and is supposed to reduce operating time. It is recommended to use mouth switch for the entire duration of neurosurgery operation [10]. However, we discovered that mouthpiece was not used frequently. This modality also does not offer a solution toward focus and zoom control, and requires extra efforts in coordinating head control.

Mouth-based control, we observed, is difficult especially for residents to hold the mouth-switch with lip or teeth to release the microscope breaks and move the microscope ocular by their head. Further, this interaction technique causes soaking the surgeon's mask by saliva, creating further discomfort and rejection. Moreover, surgeons consider using the mouthpiece 


\begin{tabular}{|l|c|c|c|c|c|c|}
\hline Modality & $\begin{array}{c}\text { Continuous } \\
\text { input }\end{array}$ & $\begin{array}{c}\text { Uninterrupted } \\
\text { operation }\end{array}$ & $\begin{array}{c}\text { Implicit } \\
\text { focus and zoom }\end{array}$ & $\begin{array}{c}\text { Clear view } \\
\text { maintenance }\end{array}$ & $\begin{array}{c}\text { Ergonomic } \\
\text { position }\end{array}$ & Aseptic contact \\
\hline Mouthpiece & $\mathrm{X}$ & - & - & - & - & - \\
\hline Pedal & $\mathrm{X}$ & - & - & - & - & - \\
\hline Voice & - & $\mathrm{X}$ & - & $\mathrm{X}$ & $\mathrm{X}$ & $\mathrm{X}$ \\
\hline Gaze & $\mathrm{X}$ & $\mathrm{X}$ & $\mathrm{X}$ & $\mathrm{X}$ & $\mathrm{X}$ & $\mathrm{x}$ \\
\hline
\end{tabular}

Table 1. Comparison of potential hands-free input interaction techniques for focus, zoom, and position control of a surgical microscope. An ' $x$ ' denotes a fulfillment of the requirement.

as a source of interruption. Finally, the quality of collaboration and teamwork are threatened because users can not talk with the OR team while mouthpiece is inside the mouth; after few movements using the mouthpiece the limitation of movement forced surgeons to anyway remove hands from the operating field and use the handgrip for changing the field of view with larger extent.

A second technique that has traditionally been used to adjust the microscope setting is a foot pedal (switch) [34, 35, 39]. This interaction has been often used to adjust magnification and focusing of a motorized microscope. In theory it is possible to use the foot pedal for adjusting all settings of the microscope, however, placing multiple-switches on a foot pedal increases the risk of error by hitting the wrong switch.

The main challenges of using foot pedal is however its poor ergonomic. Standing position in neurosurgery allows surgeons free mobility [22], however the pedal interaction technique requires surgeons to sit on a stool while manipulating the foot switch [32]. Sitting position for neurosurgeons is rare, except in certain operations, such as extracranialintracranial bypass operations thus using foot pedal in standing position cause fatigue. In our study, we have not registered any case of using foot pedal, however in other literature it has been observed that often a foot pedal moves away from the surgeon feet accidentally, which causes interruption because surgeons had to look down or ask a nurse to reposition the pedal [36].

Another possibility of hands-free interaction input is voice control. This technique has often been used in applications that are risk free due to persisting speech recognition errors. In neurosurgery OR with various naturally occurring sounds, speech, and noise recognition of speech is even more challenging. The implementation of voice control system in neurosurgery is discussed in previous work (e.g. [14, 19, 37, 41]), but most of these applications remained experimental and have never been commercially used to operate a surgical microscope [18]. The use of voice for adjusting microscope settings invites questions about how to simulate continuous commands such as zooming and focusing with a voice command [4]. Correspondingly, other questions have been raised [12] concerning the voice command delay -time to speak and recognized a voice command.

The next area of significant opportunity for hands-free interaction is gaze interaction technique. Gaze control systems have been proposed as an input device when it is not possible to operate a system with hands $[8,16,38]$. The improvements in computer vision-based techniques makes it possible to ap- ply gaze-based interactions in operating room. For example, gaze-contingent control has been recently proposed as an input device in minimally invasive surgery (MIS) $[27,33]$ and laparoscopic surgery [29].

\section{Gaze based input in micro-neurosurgery}

In an experiment in early 1990's Charleir et al. [4] developed an eye-based control for microscope. They divided the microscope view field into three command zones, namely, the peripheral zone, intermediate zone, and central zone. When gaze points were detected within the peripheral zone, the motorized microscope ocular could move to the corresponding direction with maximum speed and at the time gaze points arrived in the intermediate zone the speed of movement reduced till the gaze point was located at the central zone. Charleir et al. and other similar works explored the potentials of gazebased interaction from technical side rather than they would fully assessed the design implications for gaze-based interaction within surgical procedures and context.

Gaze input offers solution to numerous issues related to adjusting the microscope settings. The gaze-based interaction for changing the microscope setting would reduce number of interruptions, because practically it is possible to use gaze points for direct movements commands [8, 16, 38]. Moreover, we found that the current microscope auto-focus functionality is not used by the surgeons due to inaccuracy of the system. Gaze offers a way to overcome this problem by adding implicit information about where the surgeon is looking at before applying any auto-focus technique [11]. It is also feasible to use a gaze-controlled zooming system [30] in the microscope to reduce the number of interruption cause by adjusting the optical zoom.

Gaze-based interaction would give surgeons more natural control over positioning and adjusting the microscope and would maintain sterility of the operation zone. However, this rises a number of design challenges. The first concerns integration of an eye tracker into the operating microscope. To date, development of such technology has not been well investigated in contrast with other domains where eye-trackers are well established devices [5]. An eventual eye-tracker here has to be non-intrusive and maintain the standard interaction flow between surgeon and microscope.

The second methodological consideration here is the Midastouch (MT) problem, as reported in gaze-control research [15]: gaze-commands are activated by system accidentally due to the fact that it is hard to distinguish between unintentional looking and issuing a command. To match the the complexity of actual practice and to guarantee patient safety, the 
interaction system has to be error free. There are a number of ways to deal with intention recognition from gaze, including a dwell time system in which the surgeons look on the desire direction for a sufficient long time in order to interact with the microscope, or to integrate gaze-input system with other input modality. A more recent approach to MT is to use machine learning technique to detect the intentional and unintentional action before applying the interface commands [2].

\section{CONCLUSIONS}

Here we provided a detailed account of the interactions between practitioners and medical technology in the context of micro-neurosurgery routines and practices with a surgical microscope. Based on the findings, we derived a set of requirements for future input modalities to control a surgical microscope.

Current interaction techniques for positioning and adjusting the microscope during surgeries cause undesirable interruptions by forcing the surgeons to remove their hands from the operating field. In order to avoid such interruptions, the surgeons often assume uncomfortable positions and work under suboptimal conditions instead of interacting with the microscope. The use of microscope also makes the display showing a partial view from the microscope a central element for supporting the shared awareness between the surgeon and the scrub nurse.

The findings provide a first step towards designing new interaction techniques for a microscope in neurosurgical settings. We used the findings to evaluate the feasibility of several hands-free input modalities. Out of the available options, gaze-based interaction appears promising as it would allow continuous input without interruptions while maintaining sterility and clear view during the surgery. Furthermore, gaze-based interaction could be used to improve current autofocus function of the microscope and to provide additional information to first support the crucial interaction between the surgeon and the scrub nurse in this life-critical context and second support training in this area [6].

\section{ACKNOWLEDGMENTS}

We would like to thank all surgeons who participated in our experiment and Juha Jääskeläinen and Ville Leinonen for providing us opportunity to run the study and for their valuable comments.

\section{REFERENCES}

1. Aanestad, M. The camera as an actor design-in-use of telemedicine infrastructure in surgery. Computer Supported Cooperative Work (CSCW) 12, 1 (2003), $1-20$.

2. Bednarik, R., Vrzakova, H., and Hradis, M. What do you want to do next: a novel approach for intent prediction in gaze-based interaction. In Proc. of the symposium on eye tracking research and applications, ACM (2012), 83-90.

3. Bourne, S. K., Walcott, B. P., Sheth, S. A., and Coumans, J.-V. C. Neurological surgery: The influence of physical and mental demands on humans performing complex operations. Journal of Clinical Neuroscience 20, 3 (2013), 342-348.

4. Charlier, J., Sourdille, P., Behague, M., and Buquet, C. Eye-controlled microscope for surgical applications. Developments in ophthalmology 22 (1991), 154-158.

5. Duchowski, A. T. A breadth-first survey of eye-tracking applications. Behavior Research Methods, Instruments, \& Computers 34, 4 (2002), 455-470.

6. Eivazi, S., Bednarik, R., Tukiainen, M., von und zu Fraunberg, M., Leinonen, V., and Jääskeläinen, J. E. Gaze behaviour of expert and novice microneurosurgeons differs during observations of tumor removal recordings. In Proc. of the Symposium on Eye Tracking Research and Applications, ACM (2012), 377-380.

7. Fitzpatrick, G., and Ellingsen, G. A review of 25 years of cscw research in healthcare: contributions, challenges and future agendas. Computer Supported Cooperative Work 22, 4-6 (2013), 609-665.

8. Hansen, D. W., and Ji, Q. In the eye of the beholder: A survey of models for eyes and gaze. Pattern Analysis and Machine Intelligence, IEEE Transactions on 32, 3 (2010), 478-500.

9. Heath, C., Sanchez Svensson, M., Hindmarsh, J., Luff, P., and vom Lehn, D. Configuring awareness. Computer Supported Cooperative Work 11, 3-4 (2002), 317-347.

10. Hernesniemi, J., Niemelä, M., Karatas, A., Kivipelto, L., Ishii, K., Rinne, J., Ronkainen, A., Koivisto, T., Kivisaari, R., Shen, H., et al. Some collected principles of microneurosurgery: simple and fast, while preserving normal anatomy: a review. Surgical Neurology 64, 3 (2005), 195-200.

11. Hillaire, S., Lécuyer, A., Cozot, R., and Casiez, G. Using an eye-tracking system to improve camera motions and depth-of-field blur effects in virtual environments. In Virtual Reality Conference, IEEE (2008), 47-50.

12. Hinckley, K., Pausch, R., Goble, J. C., and Kassell, N. F. Passive real-world interface props for neurosurgical visualization. In Proc. of the SIGCHI conference on Human factors in computing systems, ACM (1994), 452-458.

13. Hindmarsh, J., and Pilnick, A. Knowing bodies at work: embodiment and ephemeral teamwork in anaesthesia. Organization Studies 28, 9 (2007), 1395-1416.

14. Hor, F., Desgeorges, M., Traina, M., and Cordoliani, Y. Neuronavigation: A cas system for neurosurgery. In Minimally Invasive Techniques for Neurosurgery. Springer, 1998, 175-178.

15. Jacob, R. J. The use of eye movements in human-computer interaction techniques: what you look at is what you get. ACM Transactions on Information Systems (TOIS) 9, 2 (1991), 152-169. 
16. Jacob, R. J., and Karn, K. S. Eye tracking in human-computer interaction and usability research: Ready to deliver the promises. The Mind's eye: Cognitive and Applied Aspects of Eye Movement Research (2003), 573-603.

17. Johnson, R., O’Hara, K., Sellen, A., Cousins, C., and Criminisi, A. Exploring the potential for touchless interaction in image-guided interventional radiology. In Proc. of the SIGCHI Conference on Human Factors in Computing Systems, ACM (2011), 3323-3332.

18. Kantelhardt, S. R., Finke, M., Schweikard, A., and Giese, A. Evaluation of a completely robotized neurosurgical operating microscope. Neurosurgery 72 (2013), A19-A26.

19. Koivukangas, J., Louhisalmi, Y., Alakuijala, J., and Oikarinen, J. Ultrasound-controlled neuronavigator-guided brain surgery. Journal of neurosurgery 79, 1 (1993), 36-42.

20. Kriss, T. C., and Kriss, V. M. History of the operating microscope: from magnifying glass to microneurosurgery. Neurosurgery 42, 4 (1998), 899-907.

21. Kusunoki, D. S., Sarcevic, A., Zhang, Z., and Burd, R. S. Understanding visual attention of teams in dynamic medical settings through vital signs monitor use. In Proc. of the 2013 conference on Computer supported cooperative work, ACM (2013), 527-540.

22. Lehecka, M., Laakso, A., Hernesniemi, J., and Çelik, Ö. Helsinki Microneurosurgery Basics and Tricks. M. Lehecka, A. Laakso and J. Hernesniemi, 2011.

23. McLaughlin, N., Carrau, R. L., Kelly, D. F., Prevedello, D. M., and Kassam, A. B. Teamwork in skull base surgery: An avenue for improvement in patient care. Surgical neurology international 4 (2013).

24. Mentis, H. M., O'Hara, K., Sellen, A., and Trivedi, R. Interaction proxemics and image use in neurosurgery. In Proc. of the 2012 ACM annual conference on Human Factors in Computing Systems, ACM (2012), 927-936.

25. Misra, B. K., and Chaudhuri, P. The operating microscope. Textbooks of Operative Neurosurgery (2 Vol.) (2005), 29.

26. Mitsuishi, M., Morita, A., Sugita, N., Sora, S., Mochizuki, R., Tanimoto, K., Baek, Y. M., Takahashi, H., and Harada, K. Master-slave robotic platform and its feasibility study for micro-neurosurgery. The Int. Journal of Medical Robotics and Computer Assisted Surgery 9, 2 (2013), 180-189.

27. Mylonas, G. P., Kwok, K.-W., Darzi, A., and Yang, G.-Z. Gaze-contingent motor channelling and haptic constraints for minimally invasive robotic surgery. In Medical Image Computing and Computer-Assisted Intervention-MICCAI. Springer, 2008, 676-683.

28. Nardi, B. A., Schwarz, H., Kuchinsky, A., Leichner, R., Whittaker, S., and Sclabassi, R. Turning away from talking heads: The use of video-as-data in neurosurgery. In Proc. of the INTERACT'93 and CHI'93 Conference on Human Factors in Computing Systems, ACM (1993), 327-334.

29. Noonan, D. P., Mylonas, G. P., Shang, J., Payne, C. J., Darzi, A., and Yang, G.-Z. Gaze contingent control for an articulated mechatronic laparoscope. In Biomedical Robotics and Biomechatronics (BioRob), IEEE (2010), 759-764.

30. Pomplun, M., Ivanovic, N., Reingold, E. M., and Shen, J. Empirical evaluation of a novel gaze-controlled zooming interface. In Proc. of HCI International (2001).

31. Sanchez Svensson, M., Heath, C., and Luff, P. Instrumental action: the timely exchange of implements during surgical operations. In Proceedings of the 10th European Conference on Computer Supported Cooperative Work (2007), 41-60.

32. Shimizu, S., Tanaka, O., Kondo, K., Yamazaki, T., Nakayama, K., Yamamoto, I., and Fujii, K. Inclined foot switches for surgical microscopes: A comfortable design for seated surgeons. Neurologia medico-chirurgica 51, 3 (2011), 260-262.

33. Staub, C., Can, S., Jensen, B., Knoll, A., and Kohlbecher, S. Human-computer interfaces for interaction with surgical tools in robotic surgery. In Biomedical Robotics and Biomechatronics(BioRob), IEEE (2012), 81-86.

34. Troutman, R. C. The operating microscope in ophthalmic surgery. Transactions of the American Ophthalmological Society 63 (1965), 335.

35. Uluç, K., Kujoth, G. C., and Baskaya, M. K. Operating microscopes: past, present, and future. Neurosurgical focus 27, 3 (2009), E4.

36. Van Veelen, M., Snijders, C., Van Leeuwen, E., Goossens, R., and Kazemier, G. Improvement of foot pedals used during surgery based on new ergonomic guidelines. Surgical Endoscopy And Other Interventional Techniques 17, 7 (2003), 1086-1091.

37. Wapler, M., Bräucker, M., Dürr, M., Hiller, A., Stallkamp, J., and Urban, V. A voice-controlled robotic assistant for neuroendoscopy. Studies in health technology and informatics (1999), 384-387.

38. Ware, C., and Mikaelian, H. H. An evaluation of an eye tracker as a device for computer input. In ACM SIGCHI Bulletin, vol. 17, ACM (1987), 183-188.

39. Yasargil, M. G. Microneurosurgery, Volume I. 1984.

40. Yasargil, M. G. Microsurgery: applied to neurosurgery. 2006.

41. Ying, X., Koivukangas, J., Alakuijala, J., Oikarinen, J., and Louhisalmi, Y. Design of high robust voice interface for speech activated neurosurgical workstation. In Computer Assisted Radiology/Computergestützte Radiologie. Springer, 1993, 429-434. 